\title{
Involving Users in OPAC Interface Design: Perspective from a UK Study
}

\author{
Elahe Kani-Zabihi and Gheorghita Ghinea \\ School of Information Systems Computing and Mathematics, Brunel University, Uxbridge, \\ UB8 3PH, UK \\ \{Elahe.Kani, George.Ghinea\}@brunel.ac.uk
}

\begin{abstract}
The purpose of this study was to determine user suggestions for a typical OPAC (Online Public Library Catalogue) application's functionality and features. An experiment was undertaken to find out the type of interactions features that users prefer to have in an OPAC. The study revealed that regardless of users' Information Technology (IT) backgrounds, their functionality expectations of OPACs are the same. However, based on users' previous experiences with OPACs, their requirements with respect to specific features may change. Users should be involved early in the OPAC development cycle process in order to ensure usable and functional interface.
\end{abstract}

\section{Introduction}

Electronic library catalogues over the Internet have evolved into applications called OPACs (Online Public Access Catalogues). Although there have been many investments undertaken by academic and research libraries targeting online resources, with academic libraries in the United Kingdom spending around $£ 25$ million on electronic resources [4], there are, however, still issues outstanding with respect to the usability of these systems [3]. Generally these systems have a lack of understanding of their users and have ignored users' perspectives. Hence, in designing and implementing OPACs users' abilities and experiences have to be taken into account, which forms the focus of the work reported in this paper.

\section{Background}

There is a great deal of research which targets users' interactions with OPACs' interfaces. However most existing user studies have mainly focused on OPACs' search engine's capabilities [1], [5], as well as on identifying user's retrieval methods [6], [8], so that users are able to find the information they need with minimal effort. Whilst such research disregards the fact that environmental conditions affect people's behaviour more than their personal goals [2], an even more important omission, in our opinion is that they implicitly assume that a good interface design has been executed in the first place. Nonetheless good interface design should ensure that OPACs' 
interfaces are friendly enough to deliver user satisfaction with the quality of their results first time round, irrespective of environmental conditions and any potential inhibiting influences these might exert.

A close look at how users interact with OPACs helps to understand users' needs and consequently to design these systems in a way appropriate to satisfy its users. We can go one step further by actually involving users in the design stage. Research shows that involving users in the development stage has resulted in delivering usable systems. Moreover, user involvement has resulted in user satisfaction at the end [7]. There are different approaches to this. In our study we are particularly interested in introducing elements of user-centred design and participatory design.

User-centred design methods focus on users through planning, design and development of a product since only users can provide "sufficient knowledge and understanding of the context of use". In a survey of user-centred design practice, conducted by Vredenburg et al. [12], results showed that there was improvement in product usability and that user-centred design methods are extensively used in industry. Moreover participatory methods allow all participants (users, designers, and developers) to contribute equally and influence OPAC design. Therefore, users as well as designers and developers have an important impact in the product outcome [9].

However, in involving users, irrespective of whether one employs a user-centred design method or participatory method, it is important to have users with variety of IT backgrounds, as their use of OPACs is very much dependent on their previous knowledge and experiences in IT. This was shown in a study measuring online (database) searching experiences, investigated by Hsieh-Yee [10] involving two groups of users. The first was made up of 32 professional online searchers (who had at least one year of search experience or had taken courses on online searching), whilst the second comprised 30 novice searchers (who had little or no search experience and had not taken any courses on online searching). This particular study investigated how people use their search methods to search an online database and also whether their search experience and subject knowledge had an effect on their searching methods. The conclusion of this study showed that in fact both the subject expertise and search experience had an effect on the search method employed by users.

From a related but a different perspective, a study carried by Fields et al. [11] observed librarians while helping students who did not have sufficient skills to find the information they need using the catalogue. Two librarians were videotaped and were asked to think out loud whilst they were interacting with the students. Here again, the conclusion of their study is that expert users are more successful at finding information than non-experts, thus supporting our view that OPAC design has to take into account user IT skills. The purpose of the study described in the present paper is therefore to inclusively involve users with a wide spectrum of IT abilities, by soliciting their opinions on what OPAC functionality should comprise, so that their expectations are satisfied. 


\section{Experiment}

In order to understand users' expectations and preferences we conducted an experiment observing their behaviour and interactions with OPACs. Moreover, as one of the focii of this experiment was to find out how users search for materials and what sort of difficulties they may experience, the results obtained have potentially a more general applicability than just strictly in the realm of OPACs.

\subsection{Participants}

In our study we were particularly interested in how the level of IT sophistication of users impacted the quality of suggestions for OPACs. Accordingly, 54 users (31 female and 23 male), aged between 19 and 49 years old, with a variety of IT knowledge and academic backgrounds participated in our experiment. They were grouped into three different categories (depending on his/her IT specification): novice, intermediate and advanced - with an equal number of users in each category [16].

\subsection{Procedure}

The experiment involved users interacting with OPACs from three UK universities (UniA, UniB, and UniC), all with differentiating of distinctive functionality features, users mastery of which was examined through the four tasks that they had to undertake. After each user had attempted these tasks, s/he was asked to complete a questionnaire soliciting her/his views on OPAC features and functionality.

The UniA OPAC updated its features in 2004-05, with users being now able to see more details about an item in the OPAC, such as the abstract of a book. However, in introducing new interfaces, tasks such as placing a hold on an item have arguably became more complex. To examine this, task one of our study targets exactly this facility of the UniA OPAC. As anecdotal evidence pointed to the possibility that the UniA OPAC help service is poor, in the second task of our study we asked users to find an inter-library loan service (not provided by the OPAC), and thus explore whether the support available is sufficient to guide users in this way.

As opposed to UniA, UniB allows users to search for books using the ISBN number from the OPAC home page, via a drop down menu from the search bar. Moreover the OPAC displays all books nearby the shelf of the searched item. Users are therefore able to see other books which might also be helpful to them, much in the same way as they would pick a book from its shelf in a physical library. Task three was thus allocated to UniB to find out whether users are able to use these services.

Finally, task four concerned the UniC OPAC. Services provided by this OPAC were relatively different to those used in UniA and UniB, with users having access to a rich functionality array. For instance, here users were able to save their searches; view their previous searches; add an item to 'Basket' for future use; email the content of an item to their email account; see the details of recently searched item; search for items using different category such as DVD, Books, music and etc; and finally users 
were able to clear 'Basket' and 'Previous Searches' lists by clicking on 'End session' button on the screen. Task four, as shall be shown below, explored whether users would use the whole functionality spectrum at their disposal. Specifically, the tasks were:

Task one: Search the library for a book, which contains material on Distributed Systems and Java. Once you have located such a book, place a 'hold' on it.

Task two: Find out whether you can order an article through the inter-library loan service.

Task three: Search for the book with ISBN number: 0471346098. When you find it, look for at least four other books within the same subject area (the Java programming language) on the same shelf as this book.

\section{Task four:}

Step 1 - Search for the book with title XML in a nutshell; view full details of the book; add the book to the 'Basket' so you can view it again; email the details of the book to yourself.

Step 2 - Search for The Godfather DVD; find out who stared in the movie; add it to the 'Basket'.

Step 3 - See the list of items you have added to 'Basket'.

Step 4 - See the list of searchers you have done so far.

Step 5 - Go to the result list of the search for the film The Godfather.

Step 6 - Clear all your searches to start a new search with one click.

All users were observed by the same single person, who recorded their action and their achievement. The observer also made sure each user understood the terminology used in the questionnaire and the purpose of each task given to them. Users were also asked to think out loud while interacting with OPACs.

To find out users' opinions about each task using the related OPAC, we asked them to fill in a questionnaire (Table 1) after accomplishing each task. This contained a set of five statements on which the user expressed their opinion on a five-point scale (strongly agree, agree, natural, disagree, and strongly disagree). Each statement describes whether the user is happy with the way the task is delivered by the OPAC or if the user would prefer to do the task using a physical library instead.

Table 1. OPACs' Questionnaire

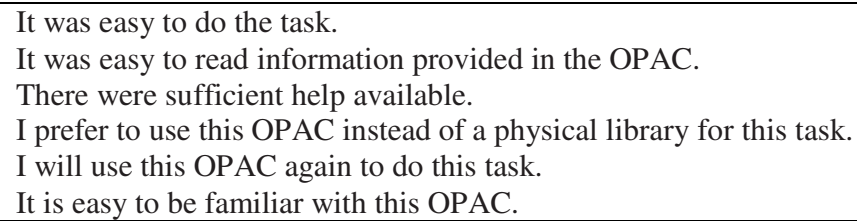

The last part of the experiment involved users replying to a questionnaire soliciting their views on OPACs in general (Table 2). The questionnaire solicited users' opinions on the features that they would prefer the OPACs to contain. 
Table 2. Users' preferences

\begin{tabular}{|c|}
\hline $\begin{array}{l}\text { From the three OPACs systems (UniA, UniB and UniC) that you have } \\
\text { used to do different tasks, which one of them was more helpful and } \\
\text { friendly to you?........... }\end{array}$ \\
\hline Specify your reason here: ..... \\
\hline
\end{tabular}

Data collected was analysed with the Statistical Package for Social Sciences (SPSS), version 11.5. The t-test and Analysis of Variance (ANOVA) were applied to identify differences, and to test potential significant differences between the three user types. The significance value used for this study was $\mathrm{p}<0.05$.

\section{Results}

\subsection{Users' Interaction with OPACs}

As mentioned above, participants were asked to use three OPACs while their interactions were observed. Almost $76 \%$ of users $(n=41)$ did not accomplish tasks correctly - and this does not exclude experienced users. Moreover, the observer noted that users often blamed their own lack of knowledge of IT when they did not achieve a task and not the application. This section describes how users achieved each task using the three OPACs.

\subsubsection{UniA OPAC}

The UniA OPAC updated its interfaces in the 2004-2005 academic year. The new version has more functions available and displays more details about a selected book. However, users who had used the catalogue in previous years commented that they prefer the old version, as it was less complicated, and tasks such as placing a hold on a book had previously been much more straightforward.

An ANOVA on our study data shows that all three different types of users said they will use UniA OPAC again ( $p=0.012, F=4.801)$ even though $31.48 \%$ of users $(\mathrm{n}=17)$, mostly novice, could not accomplish Task 1 . Fig. 1 shows the average credit given by users in their responses to the questionnaire (Table 1).

Apart from Task1-Q3 (Table 1), there was general agreement $(\mathrm{df}=53, \mathrm{p}=0.000)$ among all users as regards their ranking opinion of each task. Although all three types of users indicated it was easy to do this task, they did prefer to use a physical library for this task, especially advanced and intermediate users. This may point towards the fact that the OPAC did not satisfy users' needs and their expectations were not fulfilled. Surprisingly, all users surveyed in our study indicated that they found it easy to familiarise with the OPAC (Task1-Q6). Hence, although users were confident to 


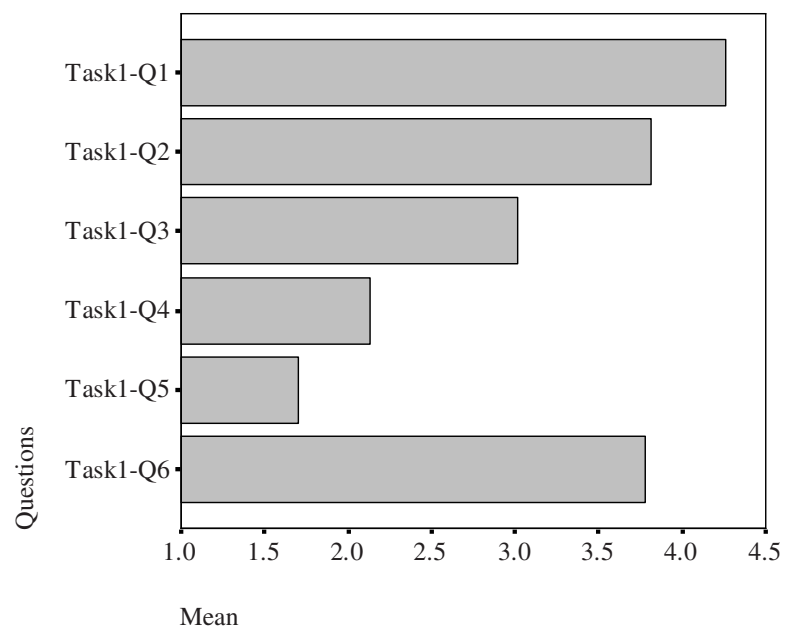

Fig. 1. Users' responses to Task 1

use the OPAC, they were not happy with the way this task could be accomplished in the OPAC.

Users, especially intermediate ones, were not in favour of using this OPAC for the task. $72.22 \%$ of novice users $(n=13)$ struggled to find the 'Place Hold' link. These users suggested that it could have been better if this option was available at the result page and this link should be placed in a more obvious place on the page. The observer noted that $18.52 \%(n=10)$ of users gave up using the Help function after their first attempt. The rest did not notice the link.

\subsubsection{UniB OPAC}

As opposed to the UniA OPAC, users were much more comfortable with using the UniB OPAC. Fig. 2 shows the average scores given by users in their responses to the questionnaire (Table 1). Apart from Q3 and Q4 (Table 1), there was general agreement $(\mathrm{df}=53, \mathrm{p}=0.000)$ among all users as regards their ranking opinion of each task. It seems that users did not all agree whether the help provided was sufficient and if they would prefer to use the OPAC in future. However, all users agreed that it was easy to use the OPAC and to read the information provided. Our results show that Intermediate users seemed to like this OPAC more than users from the other two categories.

An option to search for books by ISBN number was available under a drop down menu list on the first page of searching. Only six users (two from each category) could not find this option and hence did not find the book at the first attempt. $88.88 \%$ $(n=48)$ of users managed to find the book on the same shelf number, by clicking on the right link. The rest, however, either tried searching by the shelf number or subject name. 


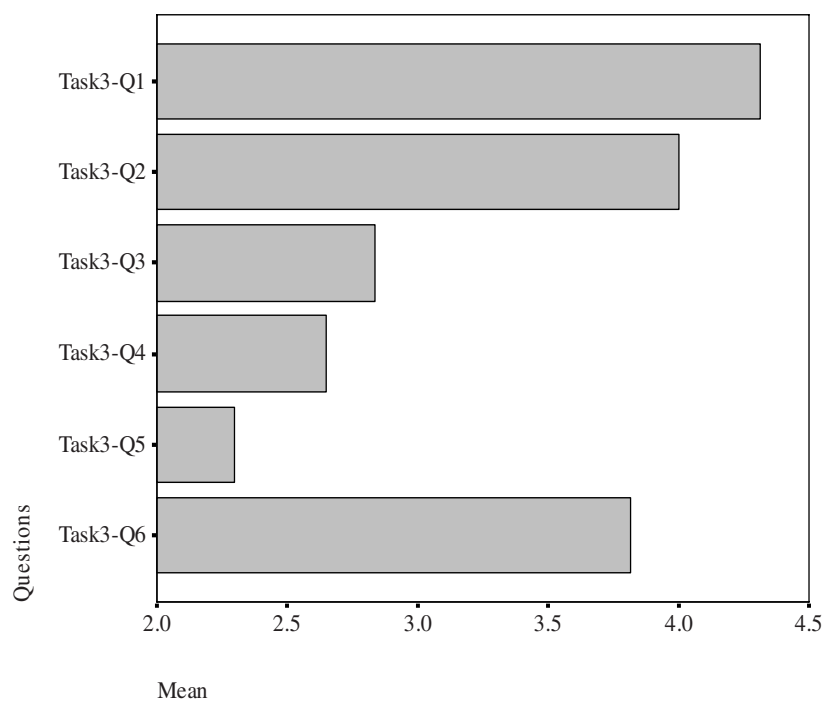

Fig. 2. Users' responses to Task 3

\subsubsection{UniC OPAC}

The result from the UniC OPAC shows that users were also happy with the interfaces of this catalogue. Fig. 3 shows the average credit given by users in their responses to the questionnaire (Table 1).

Here, we have a similar results profile to those obtained in Task 1 and Task 3. Thus, apart from Q3 (Table 1) there was general agreement $(\mathrm{df}=53, \mathrm{p}=0.000)$ among all users as regards their ranking opinion of each task.

More advanced users were in favour of UniC OPAC than the previous one. Although all users from three categories have said tasks done on this OPAC were easy, and it was easy to be familiar with the OPAC not all users accomplished their tasks correctly.

$35.19 \%$ of users $(\mathrm{n}=19)$, mainly from the novice category, could not find the 'Add to basket' link. These users were clicking on 'Basket' button instead, which was at the top of the page. One typical comment given on this function was that the terminology 'Basket' or 'Add to basket' is used for purchasing items on e-shopping websites rather than OPACs. Therefore, different phrases should be used for these functions.

$90.74 \%$ of users $(n=49)$ did not find the DVD search option under 'Subset' menu. These users searched for the DVD in much the same way as they searched for a book. It was not obvious to users that the 'Subset' button contains the list of different materials available in the OPAC e.g. DVD, Books, Journals. Some users looked for a DVD search option under the drop down menu on the first page. Only four advanced users and one intermediate user managed to find this option.

Our result indicates that the majority of users managed to view the content of the 'Basket'. Users, who did not find the option, were from all three categories. However, 


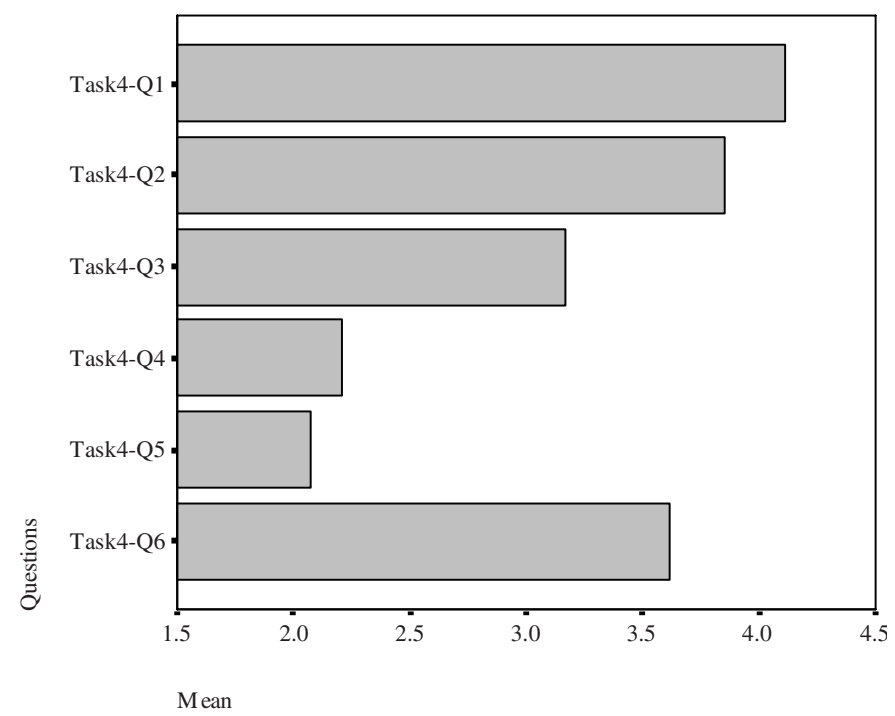

Fig. 3. Users' responses to Task 4

$35.19 \%$ of users $(n=19)$ did not click on the 'Previous Search' button which, was in the same style as 'Basket' button, this may indicate that users were not familiar with the phrase used and therefore could not make a connection between the labelled button and the task. A similar observation applies to the 'Results List' button.

Another difficult task for users was when they were required to clear their previous searches using one click. Only 29.63\% $(n=16)$ of users employed the 'End Session' button, which was designed for this purpose. These users were from all three categories. In concluding we remarked that users were generally more confident in using the UniC OPAC than the other two. Nonetheless, users commented that UniC OPAC had introduced new features, some of which were unnecessary and rather confusing. Therefore the number of people achieving Task 4 satisfactorily was low.

\section{Conclusion}

In this paper we have reported the results of a study which sought to explore the gap between users' self-reported OPAC requirements and the functionality and usability of such systems in practice. We believe that the integration of user-defined requirements of OPACs, along with other Human-Computer Interaction considerations, offer a better understanding of user perceptions and expectations with respect to OPACs and ultimately result in the design of truly user-centred OPACs. Accordingly, in our work, as opposed to previous studies ([13], [14], and [15]) in the area, which have mainly focused on information retrieval and usability issues in OPACs, we have elicited users' opinions regarding features that OPACs should have from users with a wider spectrum of IT skills, ranging from novice to advanced users. 
Results from this experiment have highlighted that users often struggle in accomplishing their tasks. We noted that novice users often blamed their own lack of knowledge of IT when they did not achieve a task and not the application. Novice users were more concerned about readability of the interfaces and receiving feedback from the application, whenever an interaction is done. Intermediate users often did not take advantage of the extra functionality of OPACs. Moreover both intermediate and advanced users highlighted the insufficient support from Help services in all three OPACs. Most of the advanced users suggested that the inter-library loan facility should be available in all OPACs.

\section{References}

[1] Babu, R.B., Tamizhchelvan, M.: An investigation into the features of OPACs in Tamil Nadu (India). Library Review 52(6), 257-267 (2003)

[2] Blandford, A., Stelmaszewska, H.: Shooting The Information Rapids. In: Vanderdonckt, Blandford, Derycke. (eds.) Ihm-HCI 2001, pp. 51-54 (2001)

[3] Bordeianu, S., Carter, C.E., Dennis, N.K.: Delivering electronic resources with Web OPACs and other Web-based tools: needs of reference librarians. Reference Service Review 28(2), 111-118 (2000)

[4] Creaser, C., Hamblin, Y.,Davies, J.E.: An assessment of potential efficiency gains through online content use. Program: electronic library and information systems 40(2), 178-189 (2006)

[5] Fox, E.A., France, R.K., Sahle, E., Daoud, A., Cline, B.E.: Development Of A Modern OPAC: From Revtolc To Marian. In: Korfhage, R., Rasmussen, E., Willett, P. (eds.) ACM Press, pp. 248-259 (June 27-July 01, 1993)

[6] Jansen, B.J., Pooch, U.: A review of web searching studies and a framework for future research. Journal of the American Society for Information Science and Technology 3(52), 235-246 (2000)

[7] Kujala, S.: User involvement: a review of the benefits and challenges. Behaviour \& Information Technology 22(1), 1-16 (2003)

[8] Tenopir, C., Hitchcock, B., Pillow, S.A.: Use and Users of Electronic Library Resources: An Overview and Analysis of Recent Research Studies. Council on Library and Information Resources (2003)

[9] Gulliksen, J., Boivie, I., Göransson, B.: Usability professionals-current practices and future development. Interacting with Computers 18(4), 568-600 (2006)

[10] Hsieh-Yee, I.: Effects of Search Experience and Subject Knowledge on the Search Tactics of Novice and Experienced Searchers. Journal of the American Society for Information Science 3(44), 161-174 (1993)

[11] Fields, B., Keith, S., Blandford, A.: Designing for Expert Information Finding Strategies. In: Fincher, S., Markopoulos, P. (eds.) People and Computers XVIII - Design for Life, pp. 89-102. Springer, Heidelberg (2004)

[12] Vredenburg, K., Mao, J., Smith, P.W., Carey, T.: A survey of User Centred Design in practice, pp. 471-478 (2002)

[13] Kan, M., Poo, D.C.C.: Detecting and supporting known item queries in online public access catalogues. In: Proceedings of the 5th ACM/IEEE-CS joint conference on Digital libraries, pp. 91-99. ACM Press, New York, USA (2005) 
[14] Agosti, M., Masotti, M.: Design of an OPAC database to permit different subject searching accesses in a multi-disciplines universities library catalogue database. In: Belkin, N., Ingwersen, P., Pejtersen, A.M. (eds.) Proceedings of the 15th annual international ACM SIGIR conference on Research and development in information retrieval, pp. 245-255 (June 21-24, 1992)

[15] Mcmullen, S.: Usability testing in a library Web site redesign project. Reference Service Review 29(1), 7-22 (2001)

[16] Kani-Z, E., Ghinea, G., Sherry, C.: Digital Libraries: What users want? Online Information Review 30(4), 395-412 (2006) 